TITLE:

\title{
Verification of reliability and validity of motion analysis systems during bilateral squat using human pose tracking algorithm
}

\section{$\operatorname{AUTHOR}(\mathrm{S}):$}

Ota, Megumi; Tateuchi, Hiroshige; Hashiguchi, Takaya; Kato, Takehiro; Ogino, Yasuhiro; Yamagata, Momoko; Ichihashi, Noriaki

\section{CITATION:}

Ota, Megumi ...[et al]. Verification of reliability and validity of motion analysis systems during bilateral squat using human pose tracking algorithm. Gait \& posture 2020, 80: 6267

\section{ISSUE DATE:}

2020-07

URL:

http://hdl.handle.net/2433/252801

\section{RIGHT:}

(c) 2020. This manuscript version is made available under the CC-BY-NC-ND 4.0 license

http://creativecommons.org/licenses/by-nc-nd/4.0/.; The full-text file will be made open to the public on $1 \mathrm{July} 2021$ in accordance with publisher's 'Terms and Conditions for Self-Archiving':; この論文は出版社版でありません。引用の際に は出版社版をご確認ご利用ください。; This is not the published version. Please cite only the published version. 


\begin{abstract}
Background: The human tracking algorithm called OpenPose can detect joint points and calculate joint angles. However, the reliability and validity of OpenPose have not been clarified yet.
\end{abstract}

Research question: Are there the enough reliability and validity of OpenPose based motion analysis?

Methods: 20 healthy young subjects participated in this study. The motion task was a bilateral squat. The joint angles of the trunk, hip, knee, and ankle were calculated using OpenPose and VICON. Kinematic measurements by three-dimensional motion analysis devices were recorded using VICON. Simultaneously, the images were taken with a digital camera from the right side. After the images were processed with OpenPose, joint angles were calculated from estimated joint points. To confirm the test-retest reliability within device, intraclass correlation coefficients [ICC $(1,3)]$ were calculated. To confirm the validity, linear regression analysis and ICC $(2,1)$ between the data obtained by OpenPose and VICON were calculated. Furthermore, the agreement between the data obtained by OpenPose and VICON was assessed by Bland-Altman analysis.

Results: ICCs $(1,3)$ of the data obtained by OpenPose and VICON were almost perfect. There were significant associations between the data obtained by OpenPose and VICON. ICCs $(2,1)$ between the data obtained by OpenPose and VICON were almost perfect or substantial for trunk, knee and ankle joints, and fair on the hip joint. There were fixed biases on knee and ankle joints, and proportional biases on trunk and hip joint.

Significance: OpenPose based motion analysis is reliable and has the advantage of being low cost and easier to operate than conventional methods. In future, to consider the clinical utility of OpenPose, it is necessary to identify the error between the true values indicating actual joint movement and data obtained by OpenPose with its correction for fixed and proportional biases.

\title{
Keywords
}

Motion analysis; Human pose tracking algorithm; OpenPose 


\section{Introduction}

Physical therapists or athletic trainers estimate abnormalities from the motions of patients and athletes and isolate problems in a clinical setting. In most clinical and sports fields these professionals perform motion analysis by observation, and analysis by observation depends on the ability of the evaluators. However, it is difficult to confirm that the data are objective (1). Therefore, in case of more detailed motion analysis, the marker-based systems such as a three-directional motion analysis system and accelerometer are used in order to gain objective data on the motions. Many kinds of research using three-dimensional motion analysis devices such as the VICON motion system or Optotrak have already been published (2-4). However, there are some disadvantages to marker-based systems. The equipment is expensive and requires time and technical skill for attaching sensors (5). Therefore, they are used in limited special environments such as hospitals and laboratories. In most clinical fields, it is therefore difficult to establish an environment where motion evaluations could be performed using marker-based systems.

OpenPose, a posture tracking algorithm that uses deep learning was recently developed and has become an important method for human posture tracking. OpenPose is a real-time system for body, foot, hand, and facial feature points (in total 135 feature points) detection on single image (6-8). Although there are few studies about applying OpenPose to motion analysis, it is possible to record each subject during motion tasks using one or two digital video cameras and analyze motions of trunk and limbs from the captured images.

OpenPose is Open Source Software and is included in the OpenCV library $(9,10)$. The license has allowed non-commercial use for free. Although commercial use requires some expenses, it could reduce costs compared to conventional motion analysis devices such as the VICON motion system. Image analyses by OpenPose only need standard digital video cameras or digital video camera-equipped tablets available on the market in addition to software. The system is easier to use, portable, and does not require a laboratory setting. If it is indeed possible to analyze motion using OpenPose, this method could be more applicable in clinical and sports fields. However, there are few preceding studies on OpenPose; the reliability and validity of its motion analysis has not been verified yet, and its value is open to dispute. Therefore, the purpose of this study was to clarify associations and agreements of motion analysis using between OpenPose with VICON during the bilateral squat, and confirm the reliability and validity of motion analysis using OpenPose. 


\section{Methods}

\subsection{Subjects}

The subjects were 20 healthy young people (16 males and 4 females; age, $26.0 \pm 3.4$ years; mean height, $169.6 \pm 6.3$ cm; mean mass, $63.4 \pm 10.4 \mathrm{~kg}$; mean body mass index, $21.2 \pm 2.5 \mathrm{~kg} / \mathrm{m}^{2}$ ). All subjects were university students. Those who had a serious medical history in the extremities and trunk were excluded. This study was approved in advance by the ethics committee of Kyoto University (approval number: R1823), and all subjects were given full written explanations before obtaining consent to participate. The GPower V.3.1.9.3 program was used to determine appropriate sample size before this study. When single regression coefficient would exceed 0.6 , which is defined as strong (11), it was found that 17 subjects must had been enrolled to have $80 \%$ power with $5 \%$ error level on two-tailed test.

OpenPose detects feature points from changes in pixels captured by the RGB camera. In the case of subjects wearing very loose or very dark-colored garments, feature points are prone to misidentification. To prevent misidentification of feature points, the subjects were asked to wear appropriately fitted light-colored garments.

\subsection{Motion task}

In each experimental trial, participants were asked to perform a bilateral squat. Data were initially collected for 3 seconds in the standing posture with their hands folded across under their chest. The subjects were then asked to execute the bilateral squat, down in 3 seconds to the maximum range so as not to hide the reflective markers at the anterior superior iliac spine, as shown in Figure 1. The angles of trunk anterior tilt and lower limb flexion were arbitrary. Finally, the subjects returned to the initial position in 3 seconds. After several practice trials, three trials were recorded.

\subsection{Data collection and processing}

Regarding the bilateral squat, peak angles of the trunk, hip, knee, and ankle joint on the right side were measured, and the changes of each angle relative to the standing position were calculated (mean value for 2 seconds).

To measure the joint angles in a conventional way, kinematic measurements by three-dimensional motion analysis devices were recorded using the VICON motion system (Vicon Nexus; Vicon Motion Systems Ltd., Oxford, England) with eight cameras at a sampling rate of $60 \mathrm{~Hz}$ and a low-pass filter with a $6 \mathrm{~Hz}$ cut off. According to the VICON Plug-in-Gait marker placement protocol, 20 reflective markers were attached to the 7th cervical vertebra, 10th thoracic vertebra, clavicle, and sternum and bilaterally to the anterior superior iliac spine, posterior superior iliac spine, lateral thigh, lateral femoral 
epicondyle, lateral shank, lateral malleolus, second metatarsal head, and calcaneus.

Kinematic measurements by two-dimensional analysis using human pose tracking algorithms were recorded using one camera-equipped tablet. The motion images were recorded using one digital video camera-equipped tablet from the right side at a sampling rate of $60 \mathrm{~Hz}$ and a low-pass filter with a $6 \mathrm{~Hz}$ cut off. The feature points of each joint were estimated using OpenPose from the captured images of subjects during the bilateral squat recorded using one digital video camera.

2.4 Data analysis

With respect to VICON, the angles of the thoracic spine segment, hip, knee and ankle joints in the sagittal plane were processed using the Vicon Clinical Manager software package. The trunk angle was measured in the global coordinates of the thoracic spine segment. With respect to OpenPose, the joint angles were measured from the estimated feature points of each joint. Figure 1 presents the placement and definition of each feature point, and the joint angles between each feature point were calculated from the obtained marker coordinates as shown in Table 1 . The mean values of three trials were calculated and used for analyses.

2.5 Statistical analysis

First, since it was confirmed that the results of the analysis of the same image twice using OpenPose were in complete agreement the test-retest reliability within device was assessed using intraclass correlation coefficients [ICC (1, 3)]. ICCs were determined as follows: $0.81-1.0$; almost perfect, $0.61-0.80$; substantial, $0.41-0.60$; moderate, $0.21-$ 0.40; fair, $0.00-0.20$; slight (12). Next, to confirm the validity, the linear regression analyses were performed by using the data obtained by OpenPose as independent variable and the data obtained by VICON as dependent variable. ICC $(2,1)$ between the data obtained by OpenPose and VICON were calculated. Furthermore, agreements between the data obtained by OpenPose and VICON were assessed by Bland-Altman analysis. The level of significance was set at 0.05 . Statistical analyses were performed using IBM SPSS Statistics, version 26 (IBM Japan Ltd., Tokyo, Japan).

\section{Results}

\subsection{Reliability}

Mean values \pm standard deviation, regression models and ICC $(1,3)$ ] for each joint angle measured using OpenPose 
and VICON are described in Table 2. ICCs $(1,3)$ of the data obtained by VICON were almost perfect (ICCs $=0.87-0.93$, $\mathrm{p}<0.01)$. Similarly, there were high ICCs $(1,3)$ of the data obtained by OpenPose (ICCs $=0.92-0.96, \mathrm{p}<0.01)$. The excellent reliability was confirmed on the joint angles measured using OpenPose.

\subsection{Validity}

Typical example on temporal changes are indicated in Figure 2. The regression models and ICC $(2,1)$ for each joint angle measured using OpenPose and VICON are described in Table 3. Regarding all joints, the data obtained by OpenPose were associated with the data obtained by VICON $\left(\mathrm{R}^{2}=0.26-0.83, \mathrm{p}<0.05\right)$. Although, ICCs $(2,1)$ between the data obtained by OpenPose and VICON were almost perfect or substantial on trunk, knee and ankle joints (ICCs $>0.60, \mathrm{p}<0.01$ ), fair on the hip joint (ICC=0.37, $\mathrm{p}<0.05$ ). Bland-Altman plot for each joint angle measured using OpenPose and VICON are provided in Figure 3. There were proportional biases on trunk and hip joint, and as the trunk angle increased, OpenPose values trended smaller than VICON values, and the hip joint angle increased, OpenPose values trended larger than VICON values as shown. Moreover, there were fixed biases on knee and ankle joints.

\section{Discussions}

In this study, the feature points of each joint were estimated using OpenPose from the captured images of subjects during bilateral squat recorded using one digital video camera, and the joint angles were measured from the estimated feature points of each joint. The main findings of this study were that there were almost perfect ICCs $(1,3)$ on OpenPose values of all joints. Moreover, there were associations between the data obtained by OpenPose and VICON on all joints. However, there were fixed biases on knee and ankle joints, and proportional biases on trunk and hip joint. This study was the first attempt to apply OpenPose to motion analysis.

In recent years, video analysis technologies, especially marker-less systems using a human pose tracking algorithm have improved tremendously. Previous studies reported significant differences between a human pose tracking algorithm called Kinect and conventional motion analysis systems. Kinect, which was released from Microsoft in 2010, consists of RGB cameras equipped with an infrared-based depth sensor and can observe movement without attaching reflective markers and without using controllers. Thereby it is possible to recognize the position and orientation of a part of the human body. In contrast to conventional motion analysis systems, the advantages of the Kinect measured motion 
analysis systems are greater convenience to operate, portability, and economical efficiency. Several studies have examined the reliability and validity of Kinect by comparing Kinect and conventional motion analysis systems, for example, VICON and Optotrak (13-19). As a result of measuring the angles of lower limbs during treadmill gait using Kinect, ICCs of peak knee flexion-swing angle and peak knee flexion-contact angle were high, whereas ICC of total ankle dorsiflexion range was moderate, and ICC of hip flexion range was slight (13). Furthermore, in the studies about gait analysis using Kinect and VICON or Optotrack, there were significant differences of hip extension, hip flexion, knee extension and flexion angle between Kinect and VICON, and correlation coefficients between Kinect and VICON were moderate on knee extension and knee flexion but weak on hip extension and hip flexion (14). As described above, there are significant differences between Kinect and conventional motion analysis systems, therefore, at the moment, Kinect could not be said to be sufficiently reliable.

Recently released OpenPose is similar to Kinect, less expensive than conventional three-dimensional motion analysis devices such as VICON, widely available, and does not require special skills and knowledge. Furthermore, OpenPose does not need makers or sensors attached to the body and needs only a standard digital camera or camera-equipped tablet. It is more convenient than conventional devices such as VICON. However, there have been no studies about the reliability and validity of the motion analysis system using OpenPose. Therefore, in this study, we compare measurements using OpenPose with those of VICON.

First, ICCs $(1,3)$ indicating the test-retest reliability within device were in almost complete agreement. At all joint angles, there were significant positive associations between the OpenPose and VICON values. However, while ICC $(2,1)$ of the trunk, knee, ankle joints were enough high, the hip joint only was fair. Moreover, there were fixed biases on knee and ankle joints, and proportional biases on trunk and hip joint. One of the reasons is, while VICON is a three-dimensional motion analysis system, OpenPose provides only two-dimensional motion data from the image captured from provided one digital camera. As a result, motions with rotation have not been measured accurately. Another reason is that there are differences in measurement methods of angles between OpenPose and VICON, thereby the measurement values of the trunk and hip joint angles were affected by the movement of the pelvis and spine. In VICON, the trunk is defined as the sagittal displacement of the thoracic segments in the global coordinates, whereas in OpenPose it is the sagittal plane angle of a straight line connecting "Neck" and "MidHip" relative to a perpendicular line to the floor passing through "Background". 
Therefore, it is considered that OpenPose has a smaller value than VICON in motions like the upper trunk flexion relative to the lower trunk. The hip joint is defined, in VICON, as the sagittal plane angle of the thigh relative to the pelvis, whereas in OpenPose, the sagittal plane angle of a straight line connecting "RHip" and "RKnee" relative to a straight line connecting "Neck" and "MidHip". Therefore, it is considered that OpenPose has a smaller value than VICON in motions like deep flexion of the hip joint with a posterior tilt of the pelvis. In the case of the motion tasks in the sagittal or frontal plane without rotation, or without spinal flexion and pelvic tilt, two-dimensional analysis using OpenPose might be enough to provide valid results. In addition, to eliminate fixed and proportional biases, it is necessary to offset the absolute value of the joint angle.

Several limitations of this study should be noted. First, there are limitations due to the features of OpenPose. The errors between the true value indicating actual joint movement and the data obtained by OpenPose or VICON have not been revealed yet. To verify the clinical utility of OpenPose in the future, it is necessary to correct the data for fixed and proportional biases, and investigate the error between the true values and the data obtained by OpenPose with offset.

Next, there are limitations due to being two-dimensional motion analysis devices. Thereby, motion tasks with rotation may reduce accuracy. It is necessary to correct fixed and proportional biases for improving validity and confirm whether it is applicable for other motion tasks. Moreover, as a limitation of this study, it has not yet been verified that OpenPose could be applied to other motion tasks such as gait. Considering the clinical utility of OpenPose, bilateral squat was adopted for the motion task in this study, and the peak angles during motion task were measured following previous studies $(13,14,17,19)$. However, it is possible to set sampling rates of the RGB cameras used in this study up to $60 \mathrm{~Hz}$, which could apply to faster motion tasks. Finally, although the number of subjects in this study was sufficient to meet the sample sizes, it was minimum. In addition, the subjects were limited to young healthy persons, and did not include the patients and the elderly with disorders. To consider the clinical utility of OpenPose in the future, it is necessary to verify the reliability and validity for the patients and the elderly.

\section{Conclusions}

The current study verified the reliability and validity of OpenPose based motion analysis during the bilateral squat.

The test-retest reliability within device were in almost complete agreement Moreover, the angles of the trunk and lower 
limb joints measured using OpenPose were associated with those angles measured using VICON on all joints. It was suggested that it should be possible to measure joint angles using OpenPose from images captured with one digital video camera. OpenPose could reduce costs and times due to the marker-less system, and be easy to use outside laboratory. However, to enhance the clinical utility of OpenPose in the future, it is necessary to consider offset for fixed and proportional biases, and investigate the error between the true value and the data obtained by OpenPose. Future research is required to verify the reliability and validity for the patients and the elderly with disorders. 


\section{References}

1. D.E. Krebs, J.E. Edelstein, S. Fishman, Reliability of observational kinematic gait analysis, Phys Ther. 65 (1985) 1027-1033. https://doi.org/10.1093/ptj/65.7.1027.

2. H. Tateuchi, Y. Koyama, H. Akiyama, K. Goto, K. So, Y. Kuroda, N. Ichihashi, Radiographic and clinical factors associated with one-leg standing and gait in patients with mild-to-moderate secondary hip osteoarthritis, Gait Posture. 49 (2016) 207-212. https://doi.org/10.1016/j.gaitpost.2016.07.018.

3. H. Tateuchi, S. Shiratori. N. Ichihashi, The effect of three-dimensional postural change on shear elastic modulus of the iliotibial band, J Electromyogr Kinesiol. 28 (2016) 137-142. https://doi.org/10.1016/j.jelekin.2016.04.006.

4. S.H. Kim, O.Y. Kwon, K.N. Park, I.C. Jeon, J.H. Weon, Lower extremity strength and the range of motion in relation to squat depth, J Hum Kinet. 7 (2015) 59-69. https://doi.org/10.1515/hukin-2015-0007.

5. R. Baker, Gait analysis methods in rehabilitation, J Neuroeng Rehab. 3 (2006) 1-10. https://doi.org/10.1186/1743-0003-3-4.

6. Z. Cao, T. Simon, S.E. Wei, Y. Sheikh. Realtime Multi-Person 2D Pose Estimation using Part Affinity Fields. Computer Vision and Pattern Recognition (2017) https://arxiv.org/abs/1611.08050.

7. Z. Cao, T. Simon, S.E. Wei, Y. Sheikh. OpenPose: Realtime Multi-Person 2D Pose Estimation using Part Affinity Fields. IEEE Transactions on Pattern Analysis and Machine Intelligence (2019) https://arxiv.org/abs/1812.08008.

8. OpenCV 4.1.1, https://opencv.org/ (accessed 18 October 2019).

9. G. Bradski, The OpenCV Library, Dr. Dobb’s Journal of Software Tools. (2000). http://www.drdobbs.com/open-source/the-opencv-library/184404319.

10. G. Hidalgo, Z. Cao, T. Simon, S.E. Wei, H. Joo, Y. Sheikh, OpenPose library. https://github.com/CMU-Perceptual-Computing-Lab/openpose.

11. J. D. Evans, Straightforward statistics for the behavioral sciences, Pacific Grove, CA: Brooks/Cole Publishing, Stamford, 1996.

12. J.R. Landis, G.G. Koch, The measurement of observer agreement for categorical data. Biometrics. 33 (1977) 159-174.

13. B.F. Mentiplay, L.G. Perraton, K.J. Bower, Y.H. Pua, R. McGaw, S. Heywood, et al., Gait assessment using the 
Microsoft Xbox One Kinect: Concurrent validity and inter-day reliability of spatiotemporal and kinematic variables, J Biomech. 48 (2015) 2166-2170. https://doi.org/10.1016/j.jbiomech.2015.05.021.

14. A. Pfister, A.M. West, S. Bronner, J.A. Noah, Comparative abilities of Microsoft Kinect and Vicon 3D motion capture for gait analysis, J Med Eng Technol. 38 (2014) 272-280. https://doi.org/10.3109/03091902.2014.909540.

15. J. Behrens, C. Pfüller, S. Mansow-Model, K. Otte, F. Paul, A.U. Brandt, Using perceptive computing in multiple sclerosis-the Short Maximum Speed Walk test, J Neuroeng Rehab. 11 (2014) 89. https://doi.org/10.1186/1743-0003-11-89.

16. R.A. Clark, K.J. Bower, B.F. Mentiplay, K. Paterson, Y.H. Pua, Concurrent validity of the Microsoft Kinect for assessment of spatiotemporal gait variables, J Biomech. 46 (2013) 2722-2725. https://doi.org/10.1016/j.jbiomech.2013.08.011.

17. B. Galna, G. Barry, D. Jackson, D. Mhiripiri, P. Olivier, L. Rochester, Accuracy of the Microsoft Kinect sensor for measuring movement in people with Parkinson's disease, Gait Posture. 39 (2014) 1062-1068. https://doi.org/10.1016/j.gaitpost.2014.01.008.

18. S. Springer, G.Y. Seligmann, Validity of the Kinect for gait assessment: A focused review, Sensors (Basel) 16 (2016) 194. https://doi.org/10.3390/s16020194.

19. X. Xu, R.W. McGorry, L.S. Chou, J.H. Lin, C.C. Chang, Accuracy of the Microsoft Kinect for measuring gait parameters during treadmill walking, Gait Posture. 42 (2015) 145-151. https://doi.org/10.1016/j.gaitpost.2015.05.002. 


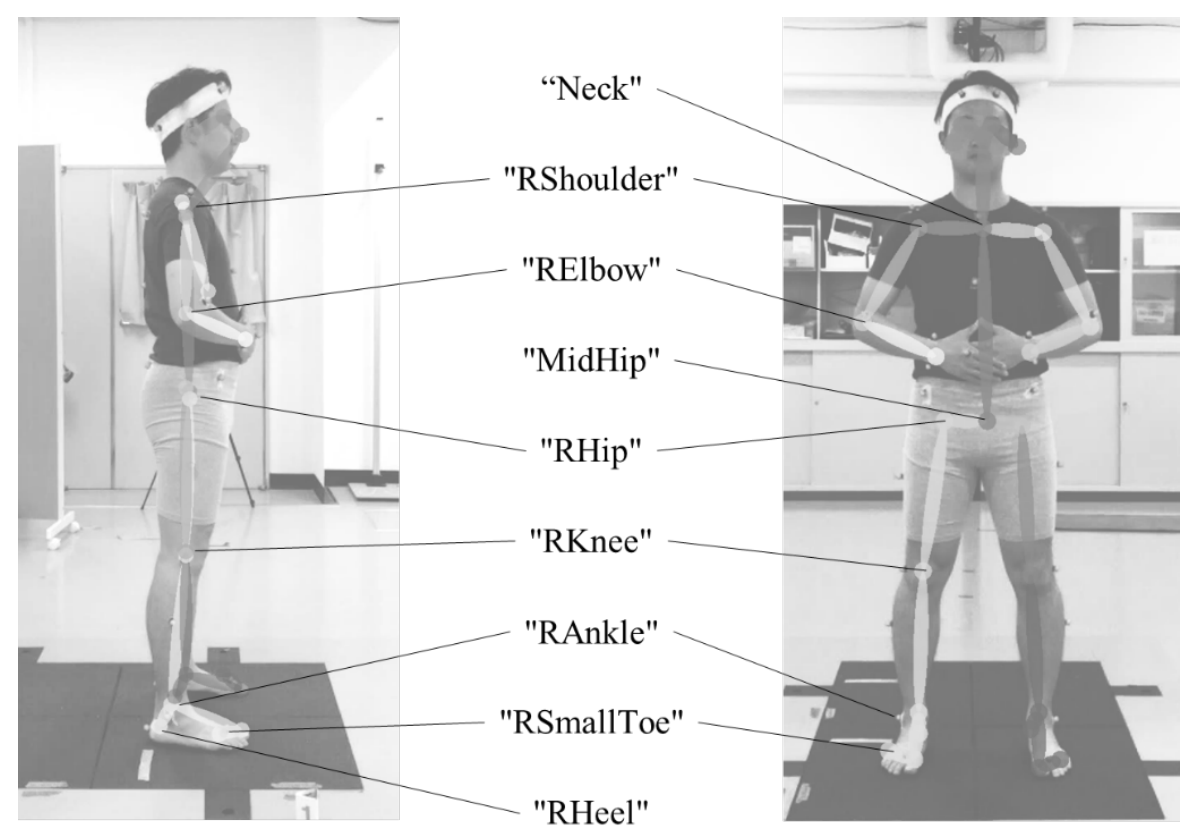

a. Standing posture with hands folded across the chest

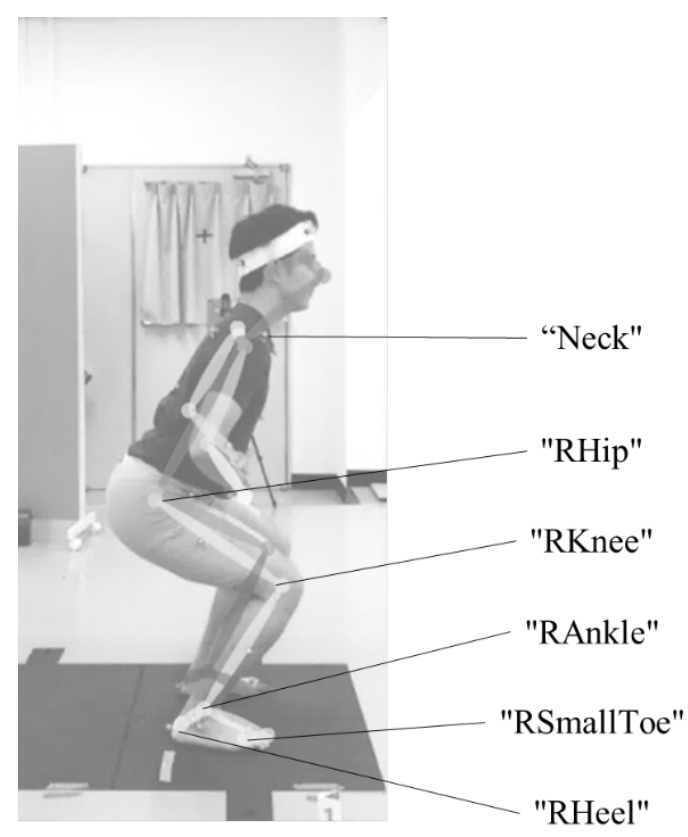

b. Bilateral squat

Figure 1. Placement and definition of each feature point in OpenPose

The figure shows the feature points estimated by OpenPose in the standing posture with the hands folded across the chest

(a) and during a bilateral squat (b).

Abbreviations: Neck-border between cervical and thoracic vertebrae; RShoulder-right shoulder joint; RElbow-right elbow joint; MidHip-center of right and left hip joints; RHip-right hip joint; RKnee-right knee joint; RAnkle-right ankle joint; RSmallToe-right 5th metatarsophalangeal joint; RHeel-right heel. 

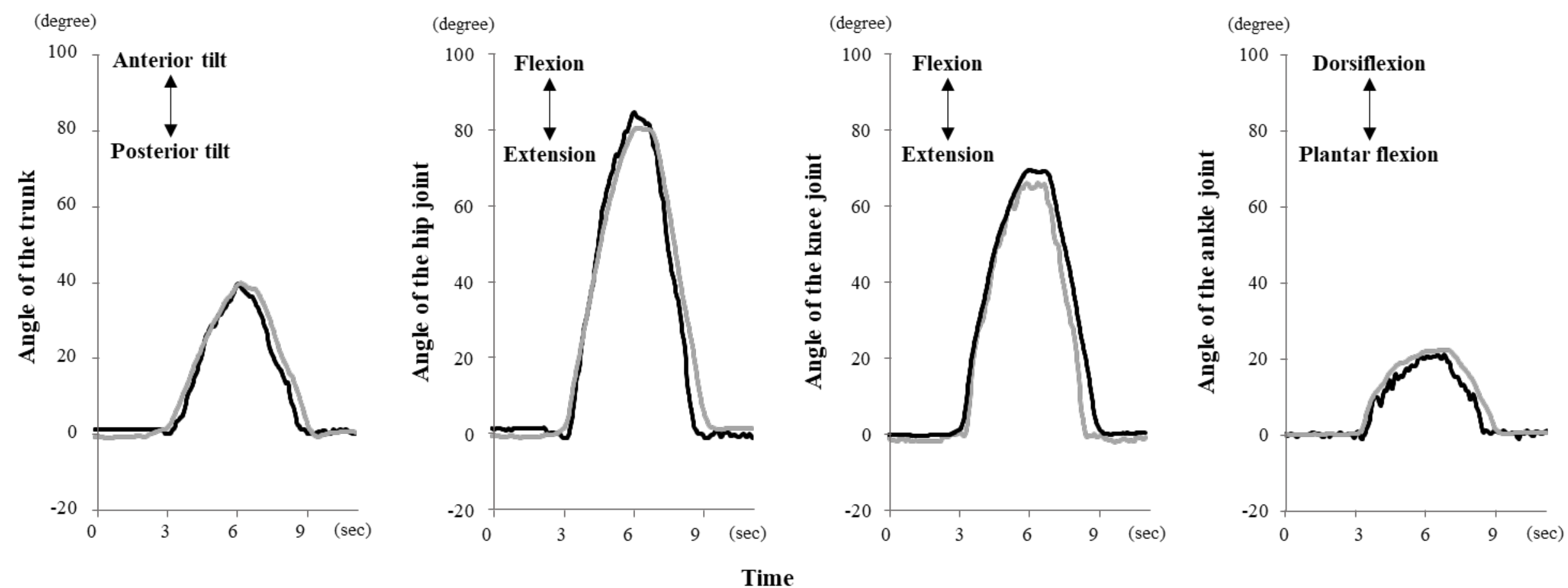

Figure 2. Typical example on temporal changes during the bilateral squat.

Foot note: Black line denotes the data obtained by OpenPose and gray lines denote the data obtained by VICON. 


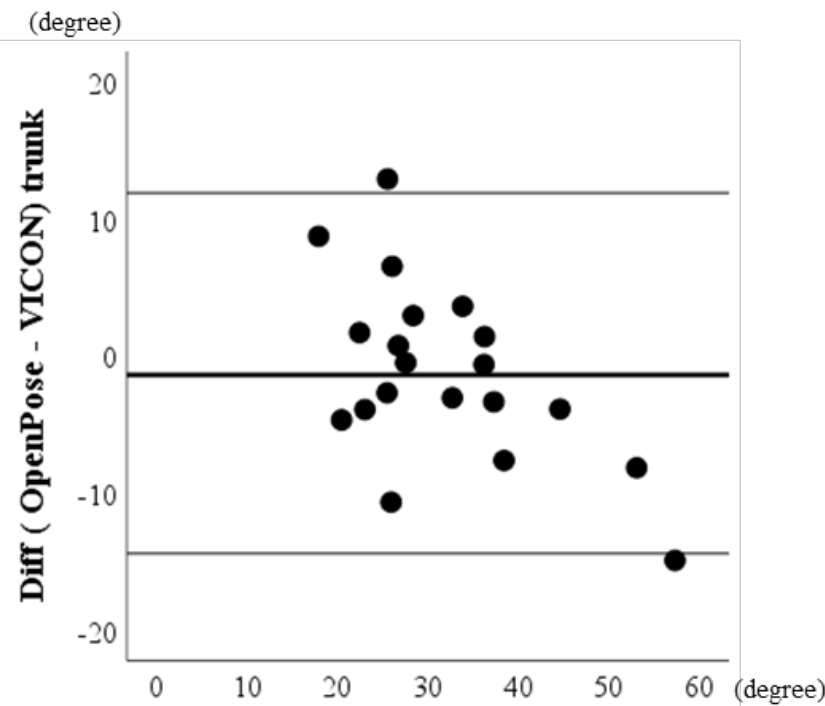

Mean (OpenPose and VICON) trunk

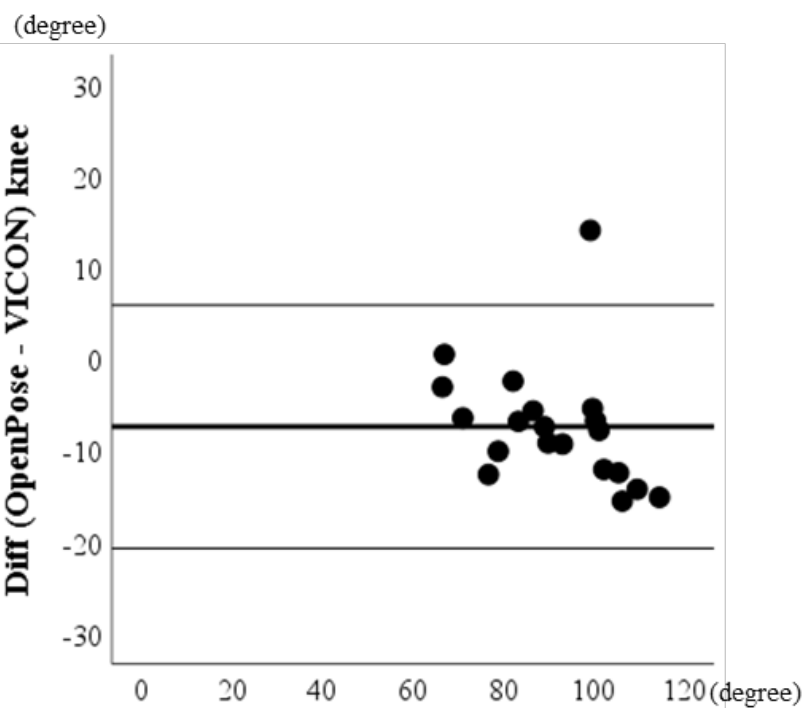

Mean (OpenPose and VICON) knee

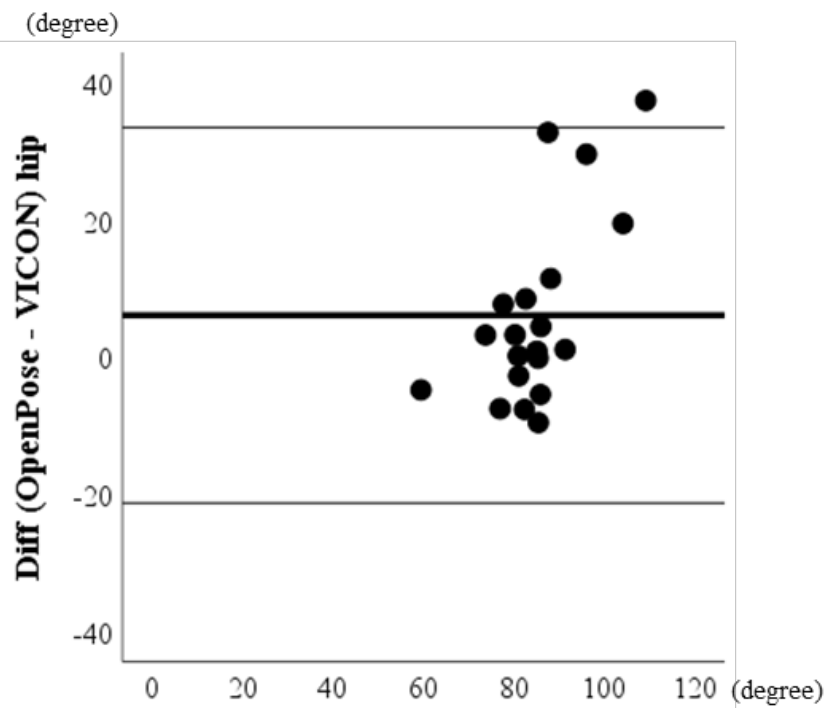

Mean (OpenPose and VICON) hip

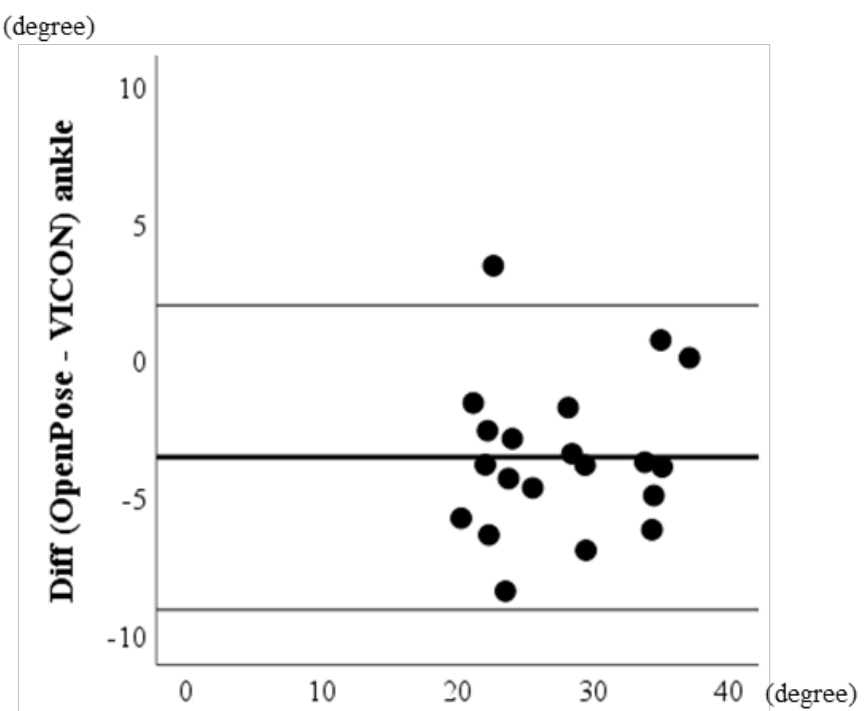

Mean (OpenPose and VICON) ankle

Figure 3. Bland-Altman plot for each joint angle measured using OpenPose and VICON.

Abbreviations: Diff (OpenPose - VICON): value measured using VICON subtracted from value measured using

OpenPose; Mean (OpenPose and VICON): mean of measurements using VICON and measurements using OpenPose.

Foot note: Thick line denotes bias (mean of difference) and thin lines denote 95\% limits of agreement (upper: mean +1.96 standard deviations of difference; lower: mean - 1.96 standard deviations of difference). 
Table 1. Axis and movement directions of each joint by OpenPose

\begin{tabular}{ll}
\hline Joint & Angle \\
\hline Trunk & The angle of a straight line connecting "Neck" and "MidHip" relative to vertical \\
Hip & $\begin{array}{l}\text { The angle of a straight line connecting "RHip" and "RKnee" relative to } \\
\text { a straight line connecting "Neck" and "MidHip" }\end{array}$ \\
Knee & The angle of "RHip", "RKnee" and "RAnkle" \\
Ankle & \begin{tabular}{l} 
The angle of a straight line connecting "RHeel" and "RSmallToe" relative to \\
\hline
\end{tabular}
\end{tabular}

Abbreviations: Neck-the border between cervical and thoracic vertebrae; MidHip-the center of right and left hip joints;

RHip-right hip joint; RKnee-right knee joint; RAnkle-right ankle joint; RSmallToe-right 5th metatarsophalangeal joint;

RHeel-right heel. 
Table 2. Mean values \pm standard deviation and the intraclass correlation coefficient [ICC $(1,3)]$ for each joint angle measured using OpenPose and VICON.

\begin{tabular}{|c|c|c|c|c|}
\hline & \multicolumn{2}{|c|}{ OpenPose } & \multicolumn{2}{|c|}{ VICON } \\
\hline & $\begin{array}{r}\text { Mean value } \pm \text { standard deviation } \\
\text { (degree) }\end{array}$ & $\begin{array}{r}\text { ICC }(1,3) \text { [95\% confidence intervals] } \\
\text { (p value) }\end{array}$ & $\begin{array}{r}\text { Mean value } \pm \text { standard deviation } \\
\text { (degree) }\end{array}$ & $\begin{array}{r}\text { ICC }(1,3)[95 \% \text { confidence intervals] } \\
\text { (p value) }\end{array}$ \\
\hline Trunk & $31.33 \pm 8.95$ & $0.95[0.88-0.98] \quad(\mathrm{p}<0.01)$ & $32.60 \pm 12.66$ & $0.92[0.84-0.97](\mathrm{p}<0.01)$ \\
\hline Hip & $88.01 \pm 15.91$ & $0.96[0.91-0.98](\mathrm{p}<0.01)$ & $81.92 \pm 7.85$ & $0.87[0.72-0.94](\mathrm{p}<0.01)$ \\
\hline Knee & $87.48 \pm 13.55$ & $0.96[0.92-0.98](\mathrm{p}<0.01)$ & $94.86 \pm 15.81$ & $0.93[0.85-0.97](\mathrm{p}<0.01)$ \\
\hline Ankle & $25.88 \pm 5.92$ & $0.92[0.83-0.97] \quad(\mathrm{p}<0.01)$ & $29.42 \pm 5.63$ & $0.90[0.79-0.96](\mathrm{p}<0.01)$ \\
\hline
\end{tabular}

Abbreviations: ICC-intraclass correlation coefficient. 
Table 3. The regression models and intraclass correlation coefficient [ICC $(2,1)]$ for each joint angle measured using OpenPose and VICON.

\begin{tabular}{|c|c|c|c|c|c|c|c|}
\hline Dependent variable & Independent variables & $\begin{array}{r}\text { Unstandardized } \\
\text { coefficients B }\end{array}$ & Constant & $\begin{array}{r}\text { 95\% confidence intervals } \\
\text { for B } \\
\text { (p value) }\end{array}$ & $\mathrm{R}^{2}$ & $\operatorname{ICC}(2,1)$ & $\begin{array}{r}\text { 95\% confidence intervals } \\
\text { for ICC }(2,1) \\
\text { (p value) }\end{array}$ \\
\hline $\begin{array}{r}\text { Trunk angle obtained } \\
\text { by VICON }\end{array}$ & $\begin{array}{r}\text { Trunk angle obtained } \\
\text { by OpenPose }\end{array}$ & 1.23 & -5.95 & $\begin{array}{r}0.89-1.58 \\
(p<0.01)\end{array}$ & 0.76 & 0.82 & $\begin{array}{r}0.61-0.93 \\
(\mathrm{p}<0.01)\end{array}$ \\
\hline $\begin{array}{r}\text { Hip angle obtained } \\
\text { by VICON }\end{array}$ & $\begin{array}{r}\text { Hip angle obtained } \\
\text { by OpenPose }\end{array}$ & 0.25 & 59.74 & $\begin{array}{r}0.04-0.46 \\
(\mathrm{p}<0.05)\end{array}$ & 0.26 & 0.37 & $\begin{array}{r}-0.03-0.68 \\
(\mathrm{p}<0.05)\end{array}$ \\
\hline $\begin{array}{r}\text { Knee angle obtained } \\
\text { by VICON }\end{array}$ & $\begin{array}{l}\text { Knee angle obtained } \\
\text { by OpenPose }\end{array}$ & 2.09 & 1.06 & $\begin{array}{r}0.82-1.30 \\
(\mathrm{p}<0.01)\end{array}$ & 0.83 & 0.80 & $\begin{array}{r}0.13-0.94 \\
\quad(\mathrm{p}<0.01)\end{array}$ \\
\hline $\begin{array}{r}\text { Ankle angle obtained } \\
\text { by VICON }\end{array}$ & $\begin{array}{r}\text { Ankle angle obtained } \\
\text { by OpenPose }\end{array}$ & 0.84 & 7.66 & $\begin{array}{r}0.62-1.06 \\
(\mathrm{p}<0.01)\end{array}$ & 0.78 & 0.75 & $\begin{array}{r}-0.00-0.93 \\
(\mathrm{p}<0.01)\end{array}$ \\
\hline
\end{tabular}

Abbreviations: ICC-intraclass correlation coefficient. 